\title{
RESPONSE OF SOIL FUNGAL COMMUNITY STRUCTURE AND DIVERSITY TO SALINE WATER IRRIGATION IN ALLUVIAL GREY DESERT SOILS
}

\author{
GuO, H. N. - HuANG, Z. J. - LI, M. Q. - MiN, W.* \\ Department of Resources and Environmental Science, Shihezi University, Shihezi, Xinjiang \\ 832003, PR China \\ (e-mails: ghnshzu@163.com; hzjshzu@163.com; lmqshzu@163.com) \\ *Corresponding author \\ e-mail: minwei555@126.com; phone: +86-139-9953-6214 \\ (Received $1^{\text {st }}$ Feb 2020; accepted $25^{\text {th }}$ May 2020)
}

\begin{abstract}
Saline water irrigation can change the soil environment, thereby influencing soil microbial processes. In this study, a nine years saline water irrigation experiment had been conducted in Shihezi, Xinjiang Province, China, to investigate the composition and diversity of the soil fungal community. Our results showed that irrigation with either brackish or saline water significantly increased soil salinity and Available Phosphorus, but reduced soil pH, SOC, TN, and Available Kalium. Saline water irrigation significantly decreased operational taxonomic units (OTUs), ACE, Chao1, and Shannon indices but increased Simpson indices. The dominant fungal phyla were Ascomycota, Mortierellomycota, Basidiomycota, Glomeromycota, and Chytridiomycota. Irrigation with either brackish or saline water significantly reduced the abundance of Mortierellomycota but increased the abundance of Ascomycota and Basidiomycota. In addition, saline water irrigation significantly decreased the relative abundance of Glomeromycota compared with fresh water irrigation, but significantly increased the relative abundance of Chytridiomycota. Redundancy analysis (RDA) results showed that salinity (ECe) was the primary factors driving the changes in soil fungal community composition. LEfSe analysis demonstrated fungal potential biomarkers decreased by saline water irrigation. These results increase our understanding of soil ecological processes in soils that are increasingly salinized.
\end{abstract}

Keywords: water salinity, environmental variables, community composition, LEfSe analysis, highthroughput sequencing

\section{Introduction}

Fresh water scarcity is a world-wide problem, particularly in arid regions where irrigation is necessary for crop production. Xinjiang Province in northwest is classified as a temperate arid climate area which is characterized by low annual precipitation, high evaporation, water shortage and soil salinization (Wang et al., 2011; Zhang et al., 2019). These factors substantially threaten the sustainability of agriculture in this region, the main agricultural water sources in Xinjiang generally contain soluble salts (Zhou et al., 2009). Saline water may be used for irrigation in areas if appropriate crop and water management practices are used (Malash et al., 2012; Mojid and Hossain, 2013). Therefore, it is important to identify best management practices for avoiding salt accumulation in the root zone when saline water is used for irrigation. Drip irrigation is widely regarded as the most promising method for saline water irrigation (Karlberg et al., 2007). Salts introduced during the early stages of saline water irrigation can be effectively leached by subsequent applications. However, even with careful management, irrigation with saline water combined with high evapotranspiration continually intensifies salinization in arid soils. Soil salinity is a critical problem in many arid and semiarid areas, leading not 
only to the deterioration of soil biophysical properties but also to declines in soil microbial biomass and metabolic efficiency. Soil salinization also affects the composition and diversity of soil microbial communities (Yu et al., 2011; Li et al., 2015a). Soil microorganisms are increasingly recognized as the most sensitive bioindicators in the soil ecosystem, and there is growing interest in managing soil microorganisms to improve the soil environment (Ludwig-Müller, 2015).

Soil microbial communities are particularly complex and dynamic, which directly participate in some important ecological processes in soil. The composition of microbial communities varies largely across environments, displaying distinct responses to changing soil environment changes (Zhang et al., 2015; Amini et al., 2016). Microbial communities directly participate in many important ecological processes in soil, including organic matter decomposition, aggregate formation, and nutrient cycling. Soil microbial diversity and community structure, soil microbial biomass, respiration and enzymatic activities have been increasingly used as relevant indicators of soil quality (Canfora et al., 2015; Srivastava et al., 2016). Decreasing soil osmotic potential with increasing soil salinity has been found to reduce not only water availability to microbes but also microbial activity and biomass (Rietz and Haynes, 2003; Yuan et al., 2007; Chowdhury et al., 2011a, b). High salinity can suppress soil microbial growth and activity (Yuan et al., 2007; Elmajdoub et al., 2014). Soil salinity over a critical level has a strong negative impact on microbial respiration (Setia et al., 2011). Jackson and Vallaire (2009) and Neubauer (2013) observed that enzyme activity is negatively correlated with soil salinity. Salinity has been implicated as a major factor regulating bacterial composition and diversity across many different habitats (Lozupone and Knight, 2007). Morrissey and Franklin (2015) found that changes in soil microbial communities may occur gradually with exposure to elevated salinity. Soil fungi are important determinants of soil fertility and ecosystem services as they participate in all biogeochemical cycles. Therefore, a salinity gradient is likely to influence fungal diversity. While its impact on bacterial communities has been thoroughly studied, the influence of soil salinity on fungal communities has been largely overlooked.

Fungal are one of the main groups of soil microorganisms, which play an important role in nutrient cycling, pollutant degradation, and ecological restoration (Yang et al., 2015). However, scientists know less about soil fungal communities than other organisms. Thus, soil fungal have been compared to "Earth's dark matter" (Jansson and Prosser, 2013). Most studies on saline soil have focused on bacterial composition and diversity. Little is known about how long-term saline water irrigation affects fungal community structure, composition, and diversity. The objective of this field experiment was to investigate the effects of irrigation water salinity on soil fungal communities diversity and composition using high throughput sequencing of the fungal internal transcribed spacer (ITS) region. We hypothesized that nine years saline water irrigation decreases soil nutrients and fungal communities diversity, changing fungal communities structure in a gray desert soil in China. Using this methodology to identify fungal taxa can elucidate the status and function of soil fungal communities in saline water-irrigated soil and quantify the effects of environmental changes on soil fungal communities. We believe this research can increase understanding about how saline water irrigation changes soil structure and function by influencing soil microbial processes. This information is useful for managing soil and saline water resources in arid and semiarid regions. 


\section{Materials and methods}

\section{Site description}

A nine years saline water irrigation field experiment was conducted at the Shihezi University Agricultural Experimental Station, Xinjiang Province, China $\left(44^{\circ} 18^{\prime} \mathrm{N}\right.$, $86^{\circ} 02^{\prime} \mathrm{E}, 450 \mathrm{~m}$ above sea level). The experiment site has a temperate continental climate. The mean annual temperature in this region is $7.0{ }^{\circ} \mathrm{C}$ with about 170 frost-free days per year. The mean annual precipitation is $210 \mathrm{~mm}$, and the mean annual potential evaporation is $1660 \mathrm{~mm}$. The groundwater table is more than $6 \mathrm{~m}$ deep. The soil at the site is an alluvial, gray desert soil, classified as a Calcaric Fluvisol in the FAO/UNESCO System. Some of the soil properties $(0-20 \mathrm{~cm}$ depth) at the start of the experiment in 2009 were as follows: bulk density, $1.33 \mathrm{~g} \mathrm{~cm}^{-3} ; \mathrm{pH}, 7.48$; electrical conductivity $\left(\mathrm{EC}_{\mathrm{e}}\right), 2.51 \mathrm{dS} \mathrm{m}^{-1}$; organic matter, $16.84 \mathrm{~g} \mathrm{~kg}^{-1}$; total nitrogen, $1.08 \mathrm{~g} \mathrm{~kg}^{-1}$; available phosphorus (AP), $25.86 \mathrm{mg} \mathrm{kg}^{-1}$; and available potassium (AK), $253 \mathrm{mg} \mathrm{kg}^{-1}$.

This experiment had been conducted in the site for a period of nine years using a completely randomized block design with three replicates of three irrigation water salinity treatments. The three treatments were (1) fresh water: noted as FW, electrical conductivities $(\mathrm{ECW})$ of the irrigation water was $0.35 \mathrm{dS} \mathrm{m}^{-1}$; (2) brackish water: noted as $\mathrm{BW}$, electrical conductivities (ECw) of the irrigation water was $4.61 \mathrm{dS} \mathrm{m}^{-1}$; (3) saline water: noted as $\mathrm{SW}$, electrical conductivities $(\mathrm{ECW})$ of the irrigation water was $8.04 \mathrm{dS} \mathrm{m}^{-}$ ${ }^{1}$. The diagram of the experimental plot layout was presented in Figure 1. The fresh water was obtained from a well in study area. The brackish or saline water were obtained by adding equal weight of $\mathrm{CaCl}_{2}$ and $\mathrm{NaCl}$ to the well water. A tank was used to store brackish or saline water and the ECw (Irrigation water Electrical conductivity) of the desired irrigation water salinity was calibrated using a conductivity meter.

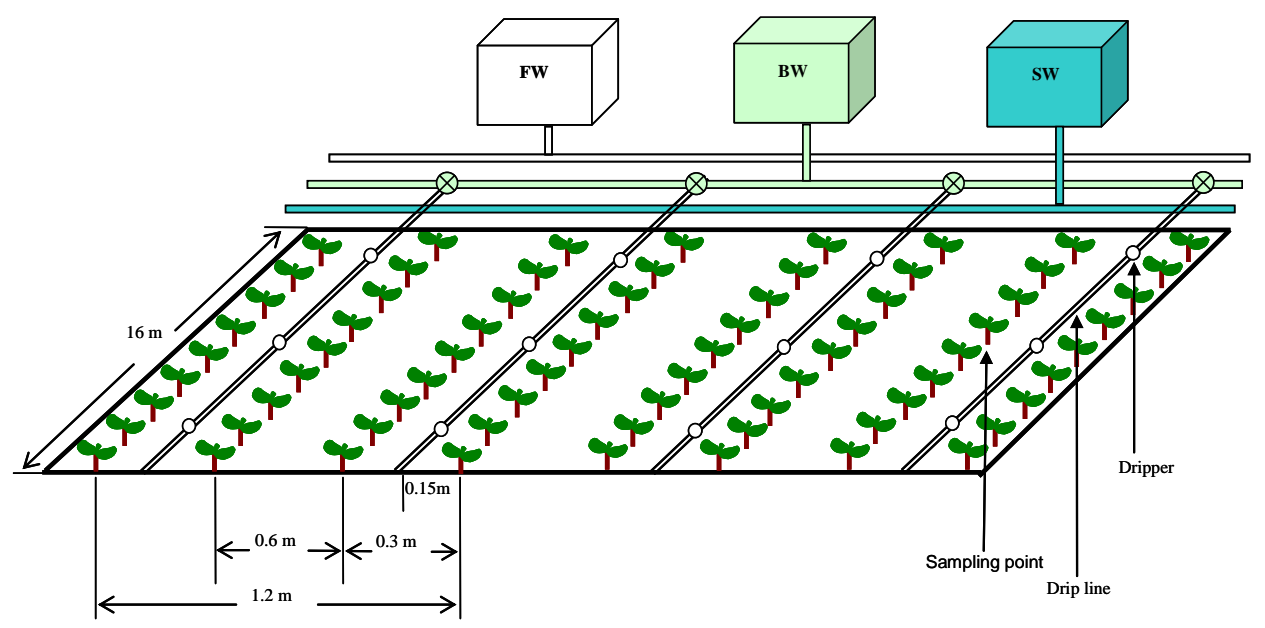

Figure 1. Diagram of the experimental plot layout

Cotton can tolerate soil salinity up to a given threshold $\left(7.7 \mathrm{dS} \mathrm{m}^{-1}\right)$, as a moderately salt-tolerant crop, cotton is considered as a model crop to understand salinity tolerance. The crop is cotton (Gossypium hirsutum L. cv Xinluzao No. 52) and usually planted in late-April and harvested in late-September on the experiment plots each year. Each plot (1.2 m wide $\times 16 \mathrm{~m}$ long) had four rows. The plots were mulched with one sheet of plastic film. Two drip irrigation lines were installed under the plastic film. Phosphorous 
$\left(\mathrm{P}_{2} \mathrm{O}_{5}\right) 105 \mathrm{~kg} \mathrm{ha}^{-1}$ and potassium $\left(\mathrm{K}_{2} \mathrm{O}\right) 60 \mathrm{~kg} \mathrm{ha}^{-1}$ were applied at planting. Nitrogen fertilizer (urea) was applied through the drip irrigation system in five equal amounts during each growing season. An irrigation experiment using different irrigation water salinity (EC of water $=0.35,4.61$, or $8.04 \mathrm{dS} \mathrm{m}^{-1}$ ) had been conducted at the study site for eight years (2009-2016) before the start of this experiment. The same irrigation amount $(450 \mathrm{~mm})$ was applied to each plot during the cotton-growing season, and the same cultivation techniques were used during the 2017 of the study.

\section{Soil sampling}

Three soil samples were collected from the $0-20 \mathrm{~cm}$ depth of each plot on July 28, 2017 (e.g. the ninth year of the study). The N fertilizer had been applied on June 27, July 4, July 12, July 19, and July 26 of that year. The soil samples were mixed and then passed through a $2 \mathrm{~mm}$ to remove stones and roots. Soil samples for DNA extraction were frozen at $-80{ }^{\circ} \mathrm{C}$. Soil samples for chemical analyses were stored at $4{ }^{\circ} \mathrm{C}$.

\section{Soil chemical analyses}

Air-dried and sieved soil subsamples were analyzed for the following physical and chemical properties: Soil salinity (ECe) was measured with a DDS-308A conductivity meter (Shanghai Precision \& Scientific Instrument Inc., Shanghai, China). Soil pH was measured in a soil-water (1:2.5, w/v) slurry using a compound electrode (PE-10; Sartorious, Germany). Soil organic carbon (SOC) was measured using the $\mathrm{K}_{2} \mathrm{Cr}_{2} \mathrm{O}_{7}-$ $\mathrm{H}_{2} \mathrm{SO}_{4}$ oxidation-reduction titration method. Soil total $\mathrm{N}$ was measured using the semimicro-Kjedahl digestion method. Available kalium (AK) and Available Phosphorus (AP) were determined using the flame photometry and molybdenum antimony resistance colorimetric method, respectively.

\section{Soil microbial analysis}

\section{DNA extraction}

Soil total bacterial DNA was extracted from $0.25 \mathrm{~g}$ of a fresh soil sample using MoBio Powersoil ${ }^{\mathrm{TM}}$ DNA Isolation kits (MoBio Laboratories, Carlsbad, CA, USA) according to the manufacturer's protocol. The concentration and purification of DNA were measured using a NanoDrop 2000 UV-vis spectrophotometer (Thermo Fisher Scientific, Waltham, MA, USA), and DNA quality was measured by $0.8 \%$ agarose gel electrophoresis. The DNA was stored at $-20^{\circ} \mathrm{C}$ before analysis.

\section{PCR amplification}

To analyze the taxonomic composition of the soil fungal community, the entire region of the $18 \mathrm{~S}$ rRNA gene was selected for amplification and subsequent highthroughput sequencing of the PCR products. The PCR amplification of the fungal internal transcribed spacer (ITS) region was performed using the forward primer ITS51737F (5'-GGAAGTAAAAGTCGTAACAAGG-3') and the reverse primer ITS22043R (5'-GCTGCGTTCTTCATCGATGC-3'). The PCR reaction system for preparing the templates contained $5 \mu \mathrm{L}$ of Q5 reaction buffer $(5 \times), 5 \mu \mathrm{L}$ of Q5 High-Fidelity GC buffer $(5 \times), 0.25 \mu \mathrm{L}$ of Q5 High-Fidelity DNA Polymerase $(5 \mathrm{U} / \mu \mathrm{L}), 2 \mu \mathrm{L}(2.5 \mathrm{mM})$ of dNTPs, $1 \mu \mathrm{L}(10 \mathrm{uM})$ of each forward and reverse primer, $2 \mu \mathrm{L}$ of DNA Template, and $8.75 \mu \mathrm{L}$ of $\mathrm{ddH}_{2} \mathrm{O}$. Thermal cycling was carried out with an initial denaturation at $95{ }^{\circ} \mathrm{C}$ 
for 3 min followed by 33 cycles of $95{ }^{\circ} \mathrm{C}$ for $30 \mathrm{~s}$, annealing at $55^{\circ} \mathrm{C}$ for $30 \mathrm{~s}$, extension at $72{ }^{\circ} \mathrm{C}$ for $45 \mathrm{~s}$, and a final extension step at $72{ }^{\circ} \mathrm{C}$ for $10 \mathrm{~min}$.

\section{Illumina MiSeq platforms sequencing}

PCR amplicons were purified with Agencourt AMPure Beads (Beckman Coulter, Indianapolis, IN) and quantified using the PicoGreen dsDNA Assay Kit (Invitrogen, Carlsbad, CA, USA). After the individual quantification step, amplicons were pooled in equal amounts, and pair-end $2 \times 300$ bp sequencing was performed using the Illlumina MiSeq platform with a MiSeq Reagent Kit v3 at Beijing Biomarker Technology Co., Ltd (Beijing, China). Each replicate was analyzed three times by high-throughput sequencing. The median of three measurements was used as the value for each replicate.

\section{Data analyses}

The one-way analysis of variance (ANOVA) and Pearson correlation analysis were conducted using SPSS statistical program (version SPSS 19.0). The difference between the groups used Tukey's test method $(\mathrm{P}<0.05)$. The sequence data were analyzed using QIIME and R packages (v3.2.0). The diversity and richness index were analyzed using Mothur software (version 1.30.1). The visualization analysis of classification and abundance used MEGAN. Linear discriminant analysis effect size (LEfSe) was performed to detect differentially abundant taxa across groups using the default parameters.

\section{Results}

\section{Soil properties}

Soil ECe, $\mathrm{pH}, \mathrm{SOC}, \mathrm{TN}, \mathrm{AK}$, and AP were significantly affected by irrigation water salinity (Table 1). Soil ECe and AP increased as water salinity increased, whereas soil $\mathrm{pH}, \mathrm{SOC}, \mathrm{TN}$, and AK decreased.

Table 1. Selected soil properties as influenced by irrigation water salinity

\begin{tabular}{c|c|c|c|c|c|c}
\hline Water salinity & ECe & pH & SOC $(\mathbf{g} / \mathbf{k g})$ & $\mathbf{T N}(\mathbf{g} / \mathbf{k g})$ & $\mathbf{A K}(\mathbf{m g} / \mathbf{k g})$ & $\mathbf{A P}(\mathbf{m g} / \mathbf{k g})$ \\
\hline FW & $3.04 \mathrm{c}$ & $7.96 \mathrm{a}$ & $10.34 \mathrm{a}$ & $0.74 \mathrm{a}$ & $252 \mathrm{a}$ & $7.24 \mathrm{c}$ \\
BW & $7.94 \mathrm{~b}$ & $7.76 \mathrm{~b}$ & $9.98 \mathrm{~b}$ & $0.70 \mathrm{~b}$ & $213 \mathrm{~b}$ & $8.92 \mathrm{~b}$ \\
SW & $11.36 \mathrm{a}$ & $7.75 \mathrm{~b}$ & $9.46 \mathrm{~b}$ & $0.64 \mathrm{c}$ & $184 \mathrm{c}$ & $11.24 \mathrm{a}$ \\
\hline
\end{tabular}

FW, BW, and SW stand for irrigation water salinities (electrical conductivity, EC) of $0.35,4.61$, and $8.04 \mathrm{dS} \mathrm{m}^{-1}$, respectively. ECe, SOC, TN, AK, and AP stand for saturation conductivity, soil organic carbon, total N, available kalium, and available phosphorus, respectively. Different letters within a column indicate significant differences at $P<0.05$

\section{Sequencing analysis and alpha diversity}

The soil fungal coverage among the soil samples was greater than $99 \%$, indicating that the depth reasonably represented actual situation of the samples (Table 2). Irrigation water salinity significantly influenced the number of OTUs. The number of OTUs was significantly less in SW than in FW and BW. The SW treatment significantly reduced the ACE and Chao1 index. However, BW and SW treatments significantly increased Simpson index. 
Table 2. Soil fungal community richness and diversity indexes as influenced by irrigation water salinity

\begin{tabular}{c|c|c|c|c|c|c|c}
\hline Water salinity & Sequence number & OTU & Coverage (\%) & ACE & Chao1 & Simpson & Shannon \\
\hline FW & $63980 \mathrm{a}$ & $426 \mathrm{a}$ & 0.9995 & $439 \mathrm{a}$ & $445 \mathrm{a}$ & $0.0526 \mathrm{c}$ & $3.86 \mathrm{a}$ \\
$\mathrm{BW}$ & $63047 \mathrm{a}$ & $435 \mathrm{a}$ & 0.9995 & $447 \mathrm{a}$ & $448 \mathrm{a}$ & $0.0627 \mathrm{~b}$ & $3.83 \mathrm{a}$ \\
SW & $63619 \mathrm{a}$ & $408 \mathrm{~b}$ & 0.9995 & $419 \mathrm{~b}$ & $421 \mathrm{~b}$ & $0.0691 \mathrm{a}$ & $3.68 \mathrm{a}$ \\
\hline
\end{tabular}

$\mathrm{FW}, \mathrm{BW}$, and $\mathrm{SW}$ stand for irrigation water salinities (electrical conductivity, EC) of $0.35,4.61$, and $8.04 \mathrm{dS} \mathrm{m}^{-1}$, respectively. OTUs, operational taxonomic units. Different letters within a column indicate significant differences at $\mathrm{P}<0.05$

Soil properties (ECe, pH, SOC, TN, AK, and AP) affected fungal community diversity indexes (Table 3). Pearson correlation coefficients showed that ACE, Chao1, and Shannon indexes were negatively correlated with ECe but positively correlated with soil SOC, TN, and AK. However, the Simpson indexes had the opposite effect.

Table 3. Correlations among selected soil properties and diversity indexes

\begin{tabular}{c|c|c|c|c|c|c}
\hline & ECe & PH & SOC & TN & AK & AP \\
\hline ACE & $-0.503 *$ & 0.243 & $0.523 *$ & $0.597 *$ & 0.459 & -0.383 \\
Chao1 & $-0.610^{*}$ & 0.400 & $0.608^{*}$ & $0.689 * *$ & $0.558^{*}$ & -0.441 \\
Simpson & $0.624^{*}$ & $-0.581 *$ & $-0.419 *$ & $-0.518^{*}$ & $-0.545^{*}$ & $0.520^{*}$ \\
Shannon & $-0.599 *$ & 0.439 & $0.509 *$ & $0.535^{*}$ & $0.550^{*}$ & $-0.625^{*}$ \\
\hline
\end{tabular}

ECe, saturation conductivity; SOC, soil organic carbon; TN, soil total nitrogen; AK, available kalium; $\mathrm{AP}$, available phosphorus. **Correlation is significant at the 0.01 level. *Correlation is significant at the 0.05 level

\section{Soil fungal community structures}

Irrigation water salinity significantly influenced the relative abundance of the dominant fungal phyla in each sample (Fig. 2). Ascomycota (55.54-63.72\%), Mortierellomycota (4.26-12.65\%), Basidiomycota (4.41-9.23\%), Glomeromycota (1.60-6.45\%), and Chytridiomycota $(2.25-9.30 \%)$ were the five most dominant phyla. Those five phyla accounted for $81.06 \%, 85.81 \%$, and $88.10 \%$ of the total relative abundance in $\mathrm{FW}, \mathrm{BW}$, and $\mathrm{SW}$, respectively. Irrigation water salinity significantly influenced the composition of the fungal communities. The BW and SW treatments significantly reduced the relative abundance of Mortierellomycota, Rotifera, and Entomophthoromycota $(P<0.05)$, but significantly increased the relative abundance of Ascomycota, Basidiomycota, and Mucoromycota $(P<0.05)$. The BW treatment significantly decreased the relative abundance of Cercozoa compared with FW, but significantly increased the relative abundance of Olpidiomycota $(P<0.05)$. The SW treatment significantly decreased the relative abundance of Glomeromycota compared with FW, but significantly increased the relative abundance of Chytridiomycota and Cercozoa $(P<0.05)$. In addition, irrigation water salinity had no significant effect on the relative abundance of Aphelidiomycota, Neocallimastigomycota, Mucoromycota, Calcarisporiellomycota, and Kickxellomycota $(P>0.05)$. 


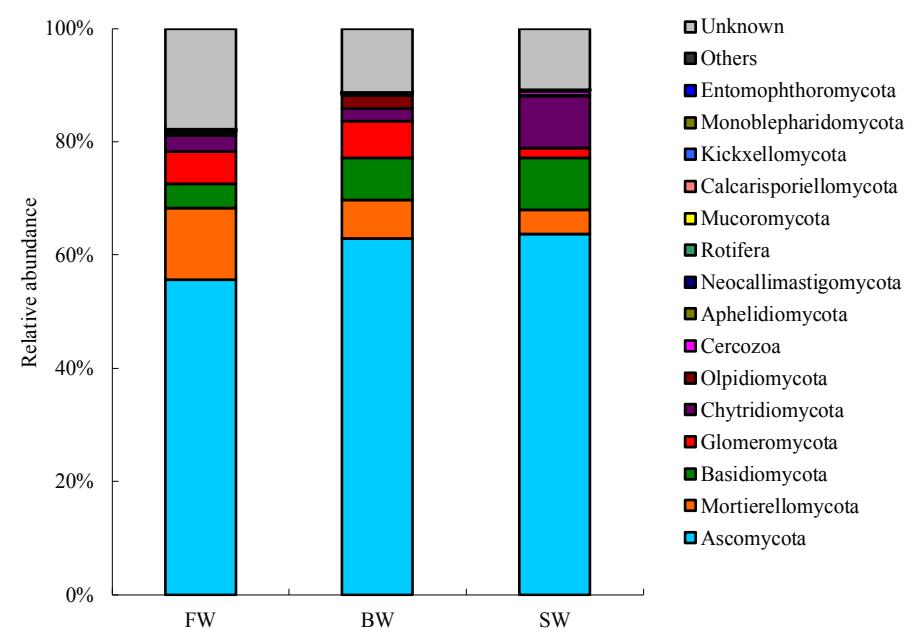

Figure 2. Relative abundance of the fifteen most common fungal phyla were influenced by irrigation water salinity. ( $F W$, fresh water $\left(0.35 d S \mathrm{~m}^{-1}\right) ; B W$, saline water $\left(4.61 \mathrm{dS} \mathrm{m^{-1 }}\right) ; S W$, saline water (8.04 $\left.\mathrm{dS} \mathrm{m}^{-1}\right)$, respectively)

Soil fungal phyla level of the different irrigation water salinity were divided into three groups (Fig. 3), indicating that fungal community in FW, BW or SW share the high similarity. A more detailed description was as follows: fresh water irrigation (FW) had the higher relative abundance of Aphelidiomycota, Monoblepharidomycota, Mortierellomycota, Entomophthoromycota, and Rotifera phyla. Howerer, irrigation with brackish water irrigation (BW) remarkably increased the relative abundance of Glomeromycota, Mucoromycota, Olpidiomycota, Calcarisporiellomycota, Kickxellomycota, Basidiomycota, Ascomycota, and Neocallimastigomycota phyla. Irrigation with saline water (SW) remarkably increased the relative abundance of Cercozoa, Anthophyta, Chytridiomycota, Basidiomycota, Ascomycota, and Neocallimastigomycota phyla.

Irrigation water salinity significantly affected the distribution of soil fungal at the genus level (Fig. 4). Chaetomium was the most abundant genus (7.54-14.79\%) followed by Mortierella (4.26-12.63\%), Curvularia (0.85-5.38\%), Fusarium (1.01$1.71 \%)$, and Verticillium $(0.35-6.19 \%)$. Combined, these five genus accounted for $23.96 \%, 26.81 \%$, and $24.06 \%$ of the total relative abundance in FW, BW, and SW, respectively. Compared with FW treatment, both BW and SW treatments significantly decreased the relative abundance of Mortierella, Entoloma, and Tetracladium $(P<0.05)$, but significantly increased the relative abundance of Curvularia and Mycosphaerella $(P<0.05)$. Compared with FW, BW significantly increased the relative abundance of Cyathus and Olpidium, SW significantly increased the relative abundance of Chaetomium, Fusarium, Vishniacozyma, and Chrysosporium $(P<0.05)$. In contrast, SW significantly reduced the relative abundance of Dominikia $(P<0.05)$. In addition, irrigation water salinity had no significant effect on the relative abundance of Verticillium, Aspergillus, and Spizellomyces $(P>0.05)$.

A heatmap showed that the relative distribution of the fungal groups varied among the treatments (Fig. 5). Each column of the heatmap shows the relative abundance of a different fungal genus in the soil sample. The columns of the heatmap are ordered according to the salinity of the soil sample. The cluster structure shows three main groups of fungal genus which share a peculiar composition and abundance among the 
sites, indicating that bacterial community in FW, BW or SW share the high similarity. A more detailed description is as follows: fresh water irrigation (FW) had the higher relative abundance of Entoloma, Cryptococcus, Talaromyces, Acremonium, Aspergillus, Pyrenochaetopsis, Dominikia, Tetracladium, Glomus, Thielaviopsis, Conidiobolus, Spizellomyces, Mortierella, Plectosphaerella, and Acrocalymma genus. Howerer, irrigation with brackish water irrigation (BW) remarkably increased the relative abundance of Stagonosporopsis, Curvularia, Alternaria, Metarhizium, Psathyrella, Verticillium, Neonectria, Olpidium, and Gibberella genus. Irrigation with saline water (SW) remarkably increased the relative abundance of Leptosphaeria, Lectera, Fusarium, Preussia, Chrysosporium, Chaetomium, Stachybotrys, Mycosphaerella, Ochroconis, and Vishniacozyma genus.

\section{RDA and correlation analysis}

The correlations between soil properties and the relative abundance of fungal phylum are given in Table 4. Four phylum (Mortierellomycota, Glomeromycota, Rotifera, and Entomophthoromycota) were negatively correlated with soil ECe and AP, however, these phylum were positively correlated with $\mathrm{pH}, \mathrm{SOC}, \mathrm{TN}$, and AK. Two phylum (Basidiomycota, Cercozoa) were positively correlated with soil ECe and AP, however, Basidiomycota and Cercozoa were negatively correlated with soil $\mathrm{pH}, \mathrm{SOC}, \mathrm{TN}$, and AK. Redundancy analysis was performed to determine the influence of various environmental parameters on fungal community structure. As shown in Figure 6, Axes 1 and 2 explained $67.93 \%$ of the variation in soil fungal community based on phylum level. The RDA plots of fungal community structure clearly show that ECe (Explain $\%=18.59 \%, p=0.046)$ is the one longer arrow. These results indicated that ECe has strong influence on soil fungal communities.

Table 4. Correlation between soil properties parameters (ECe, $p H, S O C, T N, A K$, and AP) and the relative abundance of fungal phylum

\begin{tabular}{c|c|c|c|c|c|c}
\hline & ECe & pH & SOC & TN & AK & AP \\
\hline Ascomycota & 0.521 & -0.527 & -0.646 & -0.478 & -0.604 & $0.753^{*}$ \\
Mortierellomycota & $-0.963 * *$ & $0.929 * *$ & $0.800 * *$ & $0.871 * *$ & $0.939 * *$ & $-0.819 * *$ \\
Basidiomycota & $0.733^{*}$ & $-0.690 *$ & $-0.799 * *$ & $-0.724 *$ & $-0.744 *$ & 0.625 \\
Glomeromycota & $-0.694 *$ & 0.395 & $0.746 *$ & $0.771 *$ & $0.709 *$ & $-0.763 *$ \\
Chytridiomycota & 0.34 & -0.232 & -0.110 & -0.319 & -0.245 & 0.074 \\
Olpidiomycota & -0.023 & -0.338 & 0.213 & 0.138 & 0.095 & -0.251 \\
Cercozoa & $0.668 *$ & -0.354 & $-0.764 *$ & $-0.773 *$ & $-0.695 *$ & $0.699 *$ \\
Aphelidiomycota & -0.304 & 0.476 & 0.199 & 0.198 & 0.323 & -0.331 \\
Neocallimastigomycota & 0.302 & -0.335 & -0.258 & -0.28 & -0.367 & 0.09 \\
Rotifera & $-0.754 *$ & $0.826 * *$ & 0.656 & $0.703 *$ & $0.723 *$ & -0.638 \\
Mucoromycota & 0.385 & -0.654 & -0.268 & -0.329 & -0.308 & 0.106 \\
Calcarisporiellomycota & -0.122 & -0.129 & 0.325 & 0.267 & 0.113 & -0.31 \\
Kickxellomycota & -0.097 & -0.231 & 0.194 & 0.116 & 0.164 & -0.222 \\
Monoblepharidomycota & -0.436 & 0.556 & 0.116 & 0.195 & 0.369 & -0.147 \\
Entomophthoromycota & $-0.903 * *$ & $0.957 * *$ & $0.719 *$ & $0.806 * *$ & $0.878 * *$ & $-0.718 *$ \\
\hline
\end{tabular}

ECe, saturation conductivity; SOC, soil organic carbon; TN, soil total nitrogen; AK, available kalium; AP, available phosphorus. **Correlation is significant at the 0.01 level. *Correlation is significant at the 0.05 level 


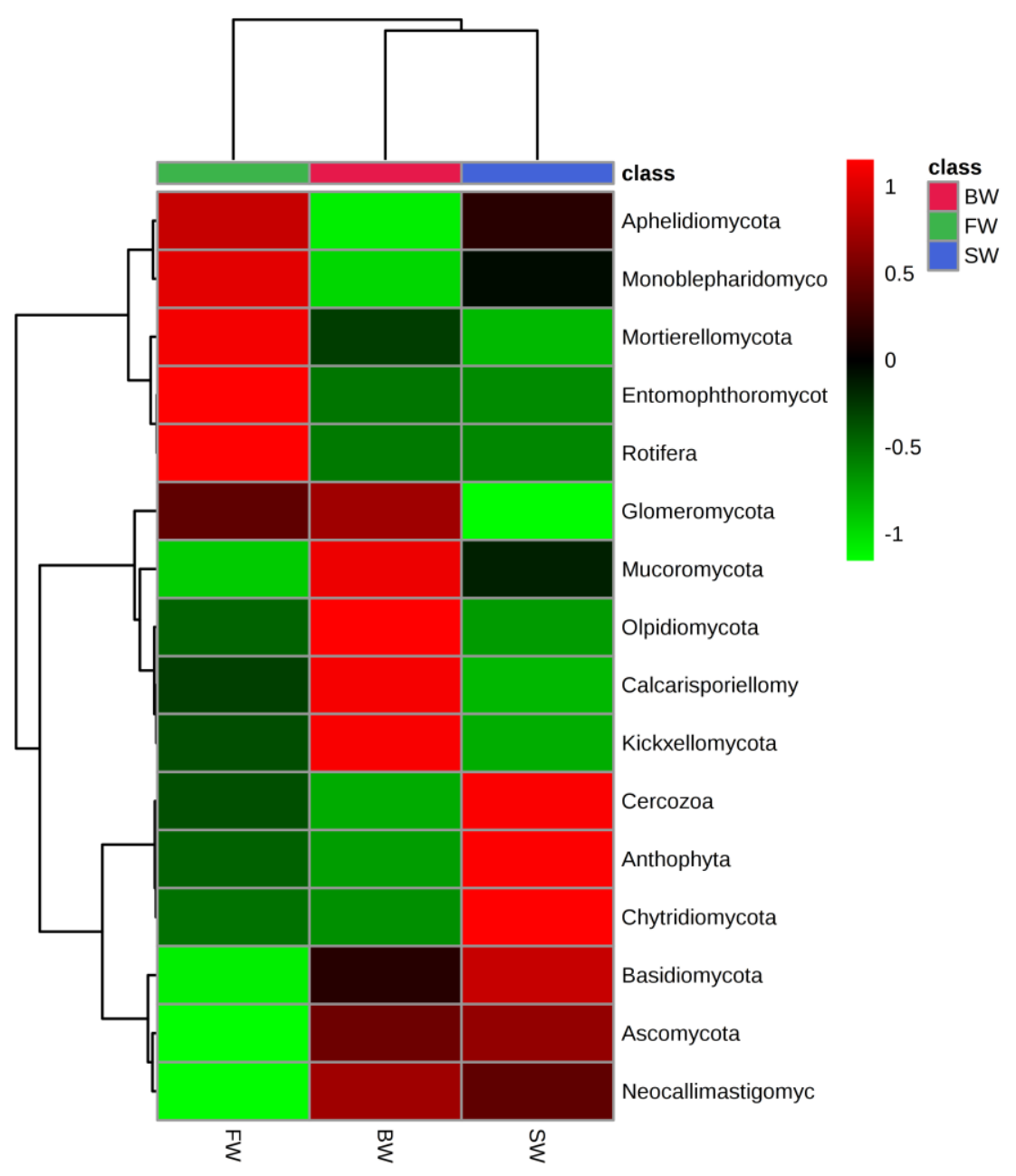

Figure 3. Heatmap showing the most relative abundance of dominant fungal phyla. The relative values are indicated by color intensity with the legend indicated at the bottom corner. $(F W$,

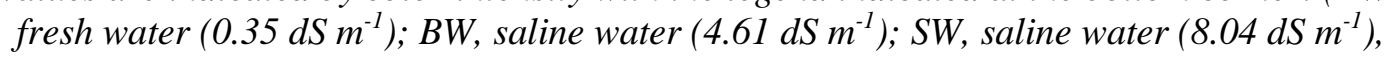
respectively)

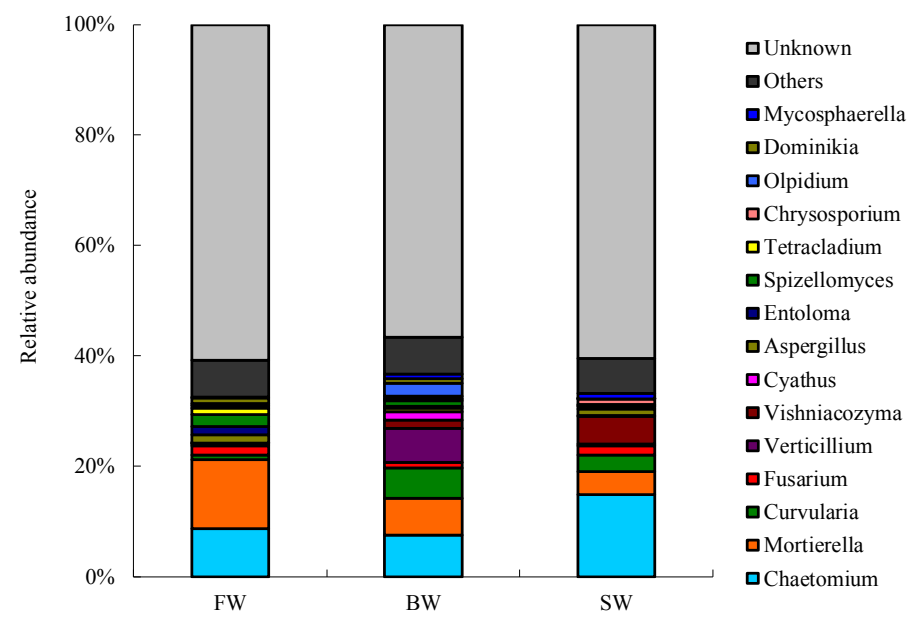

Figure 4. Relative abundance of the fifteen most common fungal genus were influenced by irrigation water salinity. ( $F W$, fresh water $\left(0.35 d S \mathrm{~m}^{-1}\right)$; $B W$, saline water $\left(4.61 \mathrm{dS} \mathrm{m^{-1 }}\right) ; S W$, saline water (8.04 $\left.\mathrm{dS} \mathrm{m}^{-1}\right)$, respectively) 


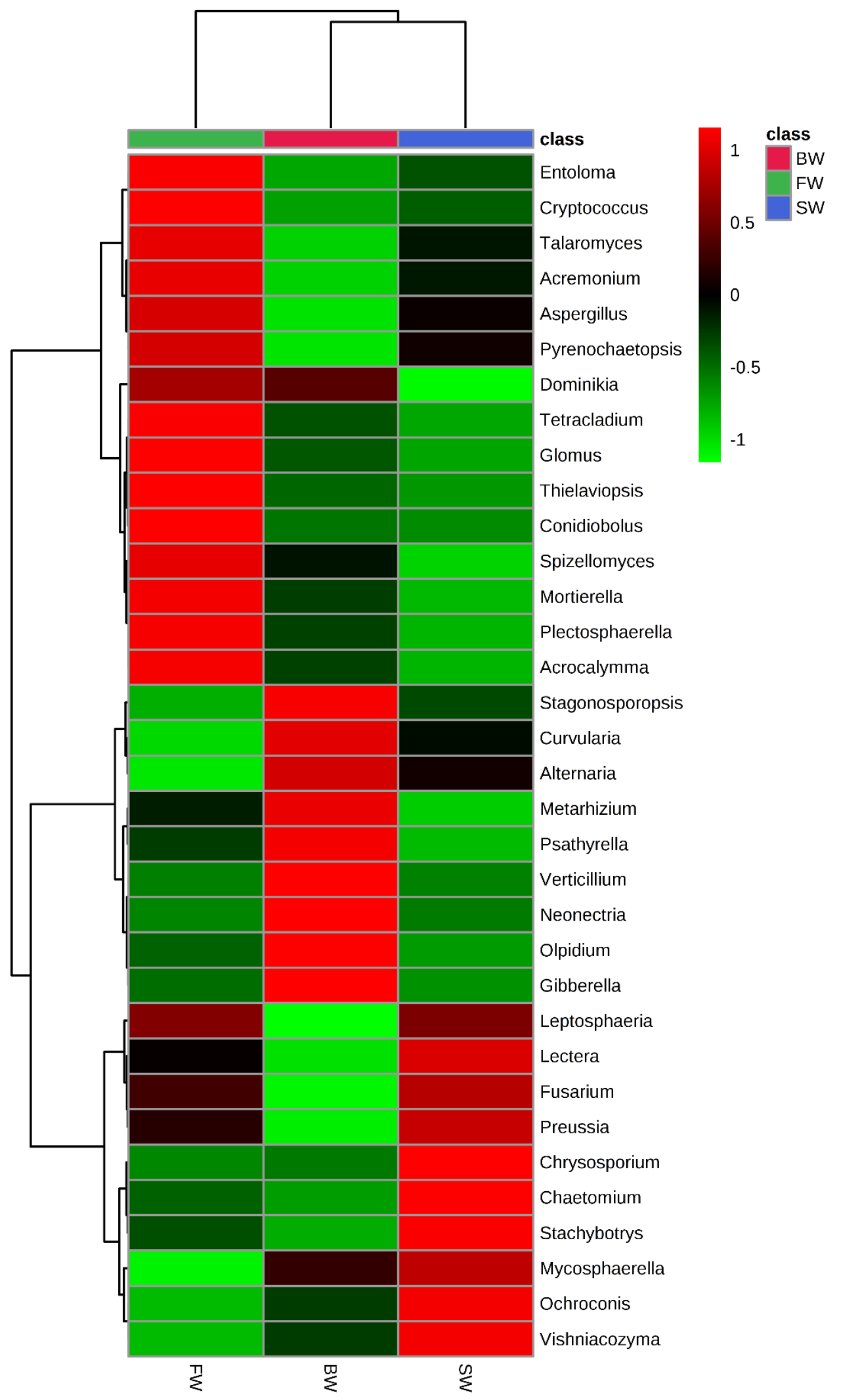

Figure 5. Heatmap showing the most relative abundance of dominant fungal genus. The relative values are indicated by color intensity with the legend indicated at the bottom corner. ( $F W$,

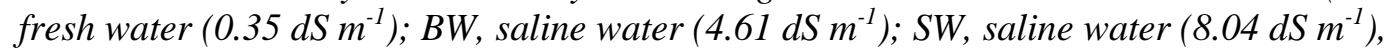
respectively) 


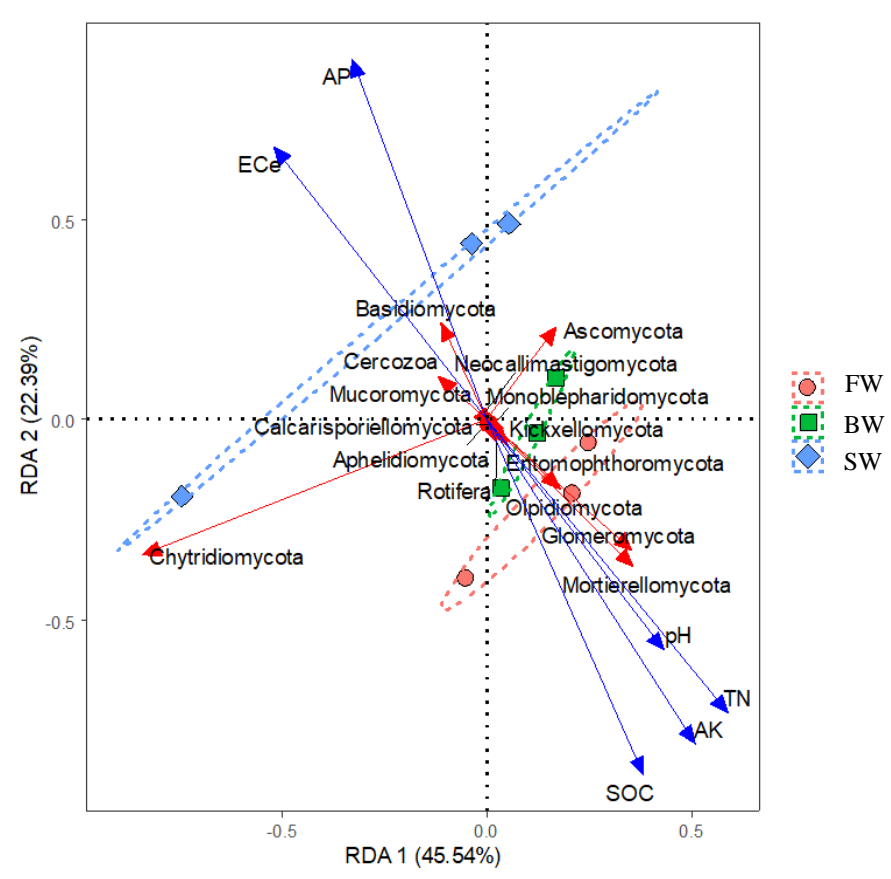

Figure 6. Redundancy analysis (RDA) results of samples based on the phylum levels. ( $F W$, fresh

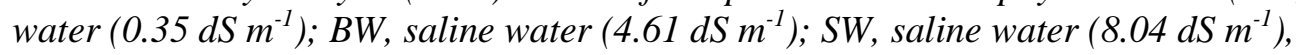
respectively; ECe, saturation conductivity; SOC, soil organic carbon; TN, soil total nitrogen; AK, available kalium; AP, available phosphorus)

The correlations between soil properties and the relative abundance of fungal genus are given in Table 5. Four genus (Mortierella, Entoloma, Tetracladium, and Dominikia) were negatively correlated with soil ECe and AP, however, these phylum were positively correlated with $\mathrm{pH}, \mathrm{SOC}, \mathrm{TN}$, and AK. Two genus (Vishniacozyma, Chrysosporium, and Mycosphaerella) were positively correlated with soil ECe and AP, however, these genus were negatively correlated with soil $\mathrm{pH}, \mathrm{SOC}, \mathrm{TN}$, and $\mathrm{AK}$. In addition, Chaetomium was negatively correlated with soil TN. The correlation of soil bacterial community structures with environmental factors were analyzed by RDA (Fig. 7). Axis 1 and axis 2 together explained $68.56 \%$ of the total variation in the compositions of soil fungal community. The RDA plots of fungal community structure clearly show that the one longer arrows were ECe (Explain $\%=25.49 \%, p=0.013)$. These results indicated that ECe has strong influence on soil fungal communities.

\section{Differential microbial composition}

A cladogram generated by LEfSe showed significant differences in taxa among the treatment groups (Fig. 8). In total, 26 bacterial clades presented significantly different among the different clades, 8 for the FW treatment, 11 for BW treatment, and 5 for the SW treatment, indicating that the potential biomarkers increased by the brackish water irrigation, however, the potential biomarkers decreased by saline water irrigation. In addition, Dothideomycetes, Pleosporales, Pleosporaceae, Curvularia, Curvularia_hawaiiensis, Auriculariales, Olpidium, Olpidiaceae, Olpidiales, Olpidiomycetes, Olpidiomycota, and Olpidium_brassicae as an indicator bacteria for BW by LEfSe analysis, Vishniacozyma_tephrensis, Tremellomycetes, Tremellales, Vishniacozyma, and Bulleribasidiaceae were indicator bacteria for SW treatment. 
Table 5. Correlation between soil properties parameters (ECe, $p H, S O C, T N, A K$, and AP) and the relative abundance of fungal genus

\begin{tabular}{c|c|c|c|c|c|c}
\hline & ECe & $\mathbf{p H}$ & SOC & TN & AK & AP \\
\hline Chaetomium & 0.551 & -0.368 & -0.639 & $-0.689 *$ & -0.562 & 0.59 \\
Mortierella & $-0.963 * *$ & $0.929 * *$ & $0.800 * *$ & $0.871 * *$ & $0.939 * *$ & $-0.820^{* *}$ \\
Curvularia & 0.532 & $-0.791 *$ & -0.289 & -0.389 & -0.484 & 0.219 \\
Fusarium & 0.128 & 0.148 & -0.383 & -0.353 & -0.212 & 0.35 \\
Verticillium & 0.059 & -0.155 & -0.004 & 0.189 & -0.07 & 0.295 \\
Vishniacozyma & $0.734 *$ & -0.584 & $-0.798 * *$ & $-0.770 *$ & $-0.746 *$ & 0.595 \\
Cyathus & 0.11 & -0.418 & 0.077 & 0.117 & -0.074 & 0.033 \\
Aspergillus & -0.349 & 0.494 & 0.134 & 0.229 & 0.293 & -0.181 \\
Entoloma & $-0.721 *$ & $0.840 * *$ & 0.534 & 0.605 & $0.681 *$ & -0.554 \\
Spizellomyces & -0.389 & 0.344 & 0.303 & 0.299 & 0.363 & -0.424 \\
Tetracladium & $-0.962 * *$ & $0.972 * *$ & $0.869 * *$ & $0.881 * *$ & $0.951 * *$ & $-0.775 *$ \\
Chrysosporium & $0.681 *$ & -0.504 & $-0.802 * *$ & $-0.806 * *$ & $-0.703 *$ & $0.705 *$ \\
Olpidium & -0.025 & -0.334 & 0.211 & 0.138 & 0.099 & -0.25 \\
Dominikia & $-0.869 * *$ & 0.645 & $0.908 * *$ & $0.880 * *$ & $0.874 * *$ & $-0.881 * *$ \\
Mycosphaerella & $0.928 * *$ & $-0.882 * *$ & $-0.847 * *$ & $-0.860 * *$ & $-0.924 * *$ & $0.710^{*}$ \\
\hline
\end{tabular}

ECe, saturation conductivity; SOC, soil organic carbon; TN, soil total nitrogen; AK, available kalium; AP, available phosphorus. **Correlation is significant at the 0.01 level. *Correlation is significant at the 0.05 level

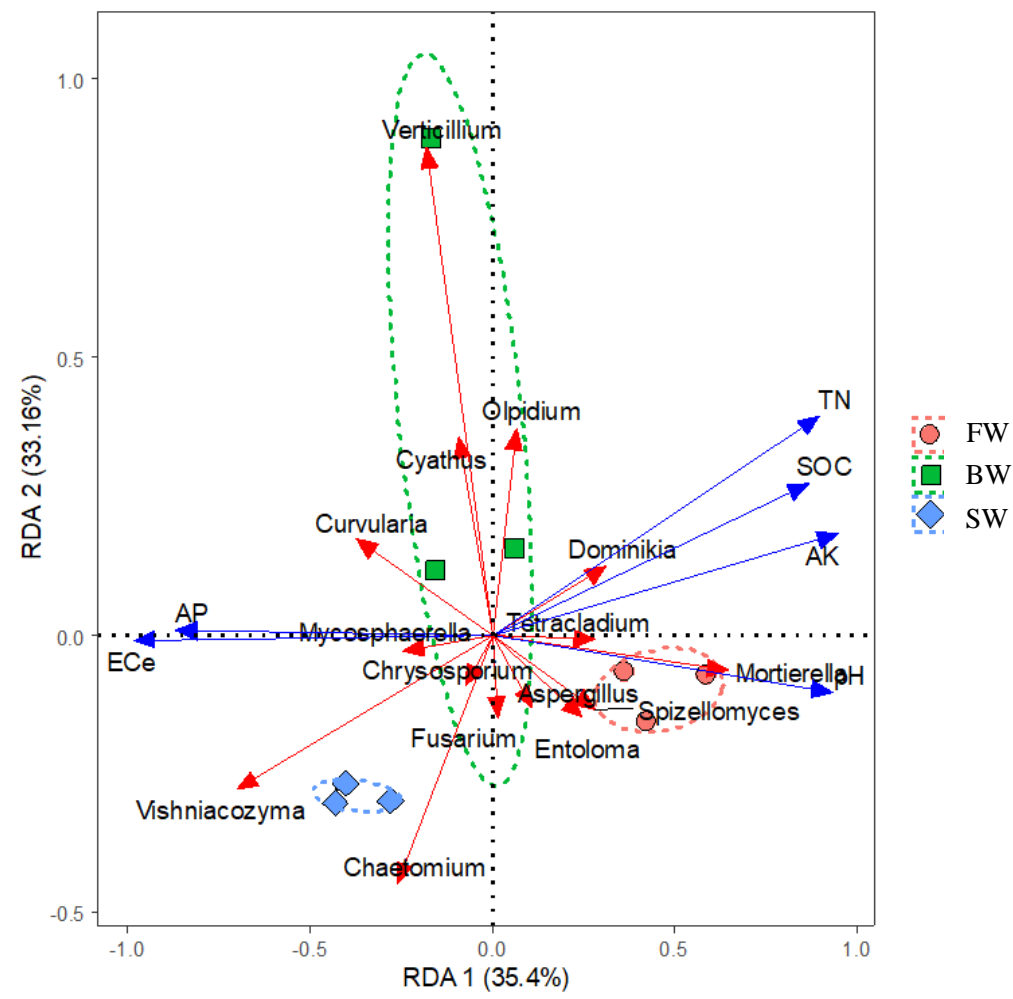

Figure 7. Redundancy analysis (RDA) results of samples based on the genus levels. ( FW, fresh

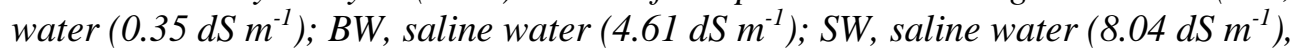
respectively; ECe, saturation conductivity; SOC, soil organic carbon; TN, soil total nitrogen; AK, available kalium; AP, available phosphorus) 
(A)

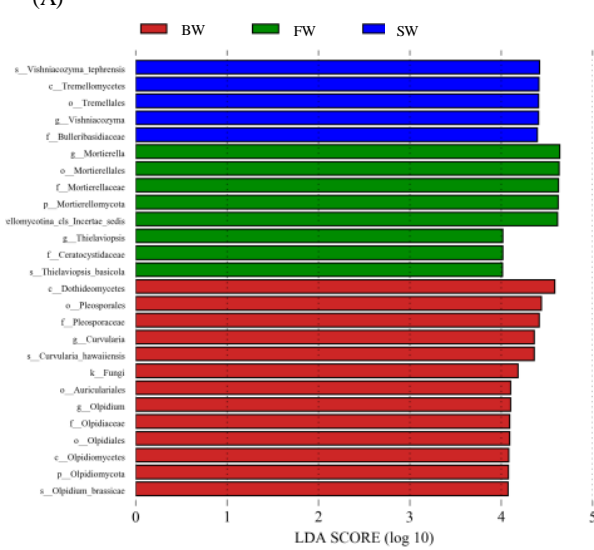

(B)

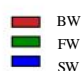

Cladogram

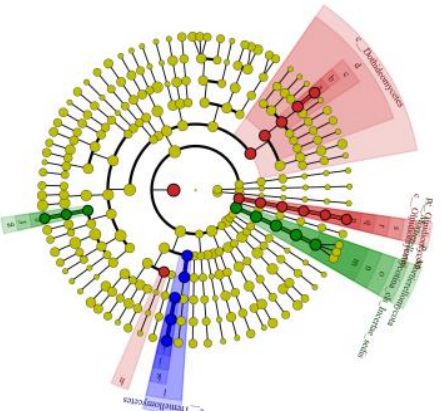

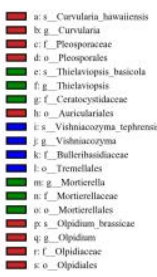

Figure 8. A linear discriminant analysis effect size (LEfSe) method identified significant differences in the abundance of fungal taxa in all of the treatments. (A) linear discriminant analysis (LDA) score; (B) Cladogram. Taxa with significantly different abundances among treatments are represented by colored dots, and from the center outward, they represent the kingdom, phylum, class, order, family, and genus levels, respectively. The colored shadows represent trends of the significantly different taxa. Each colored dot has an effect size LDA score. Only taxa meeting an LDA significance threshold of $>4$ are shown. (FW, fresh water $\left(0.35 \mathrm{dS} \mathrm{m^{-1 }}\right) ; B W$, saline water $\left(4.61 \mathrm{dS} \mathrm{m}^{-1}\right) ; S W$, saline water $\left(8.04 \mathrm{dS} \mathrm{m^{-1 }}\right)$, respectively)

\section{Discussion}

Saline water irrigation has received increasing attention, the future of irrigated agriculture will need to include the use of saline water especially in developing countries where there is extreme shortage of freshwater, particularly in arid regions (Letey and Feng, 2007). However, saline water irrigation in arid regions will load salt in the root zone, change soil physicochemical properties. The results of this study indicated that $\mathrm{BW}$ and $\mathrm{SW}$ both significantly increased soil salinity and Available Phosphorus, but reduced soil $\mathrm{pH}$, SOC, total N, Available Kalium. These changes in soil physicochemical properties may affect soil biological effectiveness.

Fungal play an important role in the soil ecosystem, which strongly influence ecosystem structure and function and are vital components of soil microbial communities with a series of important roles, such as parasitism, decomposition, pathogenesis and symbiosis (Altieri, 1999; Neher, 1999; Van der Putten et al., 2007; Aguilar-Trigueros et al., 2014). Fungal diversity and community structure were important index for evaluating the health and stability of the soil ecosystem. It is therefore necessary to increase understanding about the influence of saline water irrigation on the diversity and ecological function of soil microorganisms. Our results indicate that saline water irrigation significantly reduced the ACE and Chao1 index. This finding agrees with the findings of Cortés-Lorenzo et al. (2016) who showed that high salinity reduced fungal diversity. The effects of salinity grade on the richness (ACE, Chao1) and Shannon diversity are congruent to the negatively correlation between soil salinity and Shannon index, supporting that the diversity of fungal community decreases with increasing soil salinity, a pattern was also demonstrated by Guo and Gong (2014).

It is well known that environmental changes may affect soil fungal community composition and structure ( $\mathrm{Li}$ et al., 2015b). Many studies have found that soil 
microbial community composition may be affected by salinity (Lozupone and Knight, 2007; Llamas et al., 2008; Chowdhury et al., 2011b) because microbial genotypes differ in their tolerance of low osmotic potential (Mandeel, 2006; Llamas et al., 2008). In our study, the dominant phyla in all treatment were Ascomycota, Mortierellomycota, Basidiomycota, Glomeromycota, and Chytridiomycota. Combined, these phyla accounted for $81.06-88.10 \%$ of the relative abundance in the FW, BW, and SW treatments. In comparison, Mohamed and Martiny (2011) detected more Ascomycota (78\%), fewer Basidiomycota (6\%) and a greater number of basal fungal lineages (16\%), including Glomeromycota, Chytridiomycota and BFL1-3 in different soil (salt, brackish and freshwater marsh). Our findings are partly consistent with previous studies which found that the fungal community was mainly composed of Ascomycota, Basidiomycota, Chytridiomycota, Glomeromycota, Zygomycota, and Blastocladiomycota (MazaMárquez et al., 2016; Xu et al., 2017). Sequence abundance in this study showed that Ascomycota were the most abundant phylum. Some researchers have noted the prevalence of Ascomycota (Wang et al., 2015; Xu et al., 2017). Most Ascomycota are saprophytic fungal, which can degrade soil labile organic matter. In this study, Ascomycota phylum abundance was negatively correlated with soil SOC. In our study, BW and SW both strongly reduced the abundance of Mortierellomycota, but increased the abundance of Basidiomycota. Basidiomycota are important decomposers, which can cause wood decay and decompose wood cellulose. Wang and Guo (2016) also reported that the majority of fungal belonged to Basidiomycota in mild-salt-tolerant communities. In addition, we observed that saline water irrigation significantly decreased the relative abundance of Glomeromycota compared with fresh water irrigation, but significantly increased the relative abundance of Chytridiomycota. However, brackish water irrigation had no significant effect on the relative abundance of Glomeromycota and Chytridiomycota. The fact that fungal composition varied at all levels of taxonomic resolution along the gradient of irrigation water salinity suggests that fungal adaption to salinity (or covarying environmental parameters) occurs at many genetic scales (Martiny et al., 2009). Therefore, soil fungal communities adapted to salinity stress by adjusting their species composition.

\section{Conclusions}

In this study, we quantified the effects of nine years of saline water irrigation on soil properties and fungal community structure under drip-irrigated cotton. Saline water irrigation can change soil physicochemical properties, which thereby influence soil microbial processes. Fungal communities in the different irrigation water salinity were markedly different. Our results show that salinity (ECe) was a major environmental factor structuring the soil fungal community. saline water irrigation significantly reduced OTUs, ACE and Chao1 index. In addition, diversity of fungal community decreases with increasing soil salinity. The dominant fungal phyla were Ascomycota, Mortierellomycota, Basidiomycota, Glomeromycota, and Chytridiomycota. Saline water irrigation reduced the abundance of Mortierellomycota and Glomeromycota, but increased that of Basidiomycota, Ascomycota and Chytridiomycota were not significantly affected by saline water irrigation. In addition, the dominant fungal genus were Chaetomium, Mortierella, Curvularia, Fusarium, and Verticillium. Statistical analysis (LEfSe) demonstrated that fungal potential biomarkers decreased by saline water irrigation. The results of this study increase understanding about (i) the effects of 
irrigation water salinity on fungal community structure under irrigated cotton and (ii) the role of environmental variability as a predictor of fungal community composition. In addition, saline irrigation will increase soil salinity, thereby affecting soil $\mathrm{N}$ transformations. In the future, we should focus on the effect of saline water irrigation on soil nitrogen transformation and its key microbiological process, providing a theoretical basis for the rational use of saline water resources in arid regions and the effective management of $\mathrm{N}$ and the regulation of the $\mathrm{N}$ cycle.

Acknowledgments. This work was jointly funded by The National Natural Science Foundation of China [41661055], the Youth Innovation Talent Cultivation Program of Shihezi University [CXRC201706].

\section{REFERENCES}

[1] Aguilar-Trigueros, C. A., Powell, J. R., Anderson, I. C., Antonovics, J., Rillig, M. C. (2014): Ecological understanding of root-infecting fungal using trait-based approaches. Trends in Plant Science 19(7): 432-438.

[2] Altieri, M. A. (1999): The Ecological Role of Biodiversity in Agroecosystems. - In: Paoletti, M. G. (ed.) Invertebrate Biodiversity as Bioindicators of Sustainable Landscapes. Elsevier, Amsterdam, pp. 19-31.

[3] Amini, S., Ghadiri, H., Chen, C., Marschner, P. (2016): Salt-affected soils, reclamation, carbon dynamics, and biochar: a review. - Journal of Soils and Sediments 16(3): 939953.

[4] Canfora, L., Malusà, E., Salvati, L., Renzi, G., Petrarulo, M. C., Benedetti, A. (2015): Exploring short-term Impact of two liquid organic fertilizers on Solanum lycopersicum L. rhizosphere Eubacteria and Archaea diversity. - Applied Soil Ecology 88: 50-59.

[5] Chowdhury, N., Marschner, P., Burns, R. (2011a): Response of microbial activity and community structure to decreasing soil osmotic and matric potential. - Plant and Soil 344(1-2): 241-254.

[6] Chowdhury, N., Marschner, P., Burns, R. G. (2011b): Soil microbial activity and community composition: impact of changes in matric and osmotic potential. - Soil Biology and Biochemistry 43(6): 1229-1236.

[7] Cortés-Lorenzo, C., González-Martínez, A., Smidt, H., González-López, J., Rodelas, B. (2016): Influence of salinity on fungal communities in a submerged fixed bed bioreactor for wastewater treatment. - Chemical Engineering Journal 285: 562-572.

[8] Elmajdoub, B., Barnett, S., Marschner, P. (2014): Response of microbial activity and biomass in rhizosphere and bulk soils to increasing salinity. - Plant and Soil 381(1-2): 297-306.

[9] Guo, X., Gong, J. (2014): Differential effects of abiotic factors and host plant traits on diversity and community composition of root-colonizing arbuscular mycorrhizal fungi in a salt-stressed ecosystem. - Mycorrhiza 24(2): 79-94.

[10] Jackson, C. R., Vallaire, S. C. (2009): Effects of salinity and nutrients on microbial assemblages in Louisiana wetland sediments. - Wetlands 29(1): 277-287.

[11] Jansson, J. K., Prosser, J. I. (2013): Microbiology: the life beneath our feet. - Nature 494(7435): 40.

[12] Karlberg, L., Rockström, J., Annandale, J. G., Steyn, J. M. (2007): Low-cost drip irrigation-A suitable technology for southern Africa? An example with tomatoes using saline irrigation water. - Agricultural Water Management 89(1): 59-70.

[13] Letey, J., Feng, G. L. (2007): Dynamic versus steady-state approaches to evaluate irrigation management of saline waters. - Agricultural Water Management 91: 1-10. 
[14] Li, C. J., Lei, J. Q., Zhao, Y., Xu, X. W., Li, S. Y. (2015a): Effect of saline water irrigation on soil development and plant growth in the Taklimakan Desert Highway shelterbelt. - Soil and Tillage Research 146: 99-107.

[15] Li, Y. J., Wang, H., Zhao, J. N., Huangfu, C. H., Yang, D. L. (2015b): Effects of tillage methods on soil physicochemical properties and biological characteristics in farmland: a review. - The Journal of Applied Ecology 26(3): 939-948.

[16] Llamas, D. P., de, Cara, Gonzalez, M., Gonzalez, C. I., Lopez, G. R., Marquina, J. T. (2008): Effects of water potential on spore germination and viability of Fusarium species. - Journal of Industrial Microbiology \& Biotechnology 35(11): 1411-1418.

[17] Lozupone, C. A., Knight, R. (2007): Global patterns in bacterial diversity. - Proceedings of the National Academy of Sciences 104(27): 11436-11440.

[18] Ludwig-Müller, J. (2015): Bacteria and fungi controlling plant growth by manipulating auxin: balance between development and defense. - Journal of Plant Physiology 172: 412.

[19] Malash, N. M., Ali, F. A., Fatahalla, M. A., Hatem, M. K., Tawfic, S. (2012): Response of tomato to irrigation with saline water applied by different irrigation methods and water management strategies. - International Journal of Plant Production 2: 101-116.

[20] Mandeel, Q. A. (2006): Biodiversity of the genus Fusarium in saline soil habitats. Journal of Basic Microbiology 46(6): 480-494.

[21] Martiny, A. C., Tai, A. P., Veneziano, D., Primeau, F., Chisholm, S. W. (2009): Taxonomic resolution, ecotypes and the biogeography of Prochlorococcus. Environmental Microbiology 11(4): 823-832.

[22] Maza-Márquez, P., Vilchez-Vargas, R., Kerckhof, F. M., Aranda, E., González-López, J., Rodelas, B. (2016): Community structure, population dynamics and diversity of fungal in a full-scale membrane bioreactor (MBR) for urban wastewater treatment. - Water Research 105: 507-519.

[23] Mohamed, D. J., Martiny, J. B. (2011): Patterns of fungal diversity and composition along a salinity gradient. - The ISME Journal 5(3): 379.

[24] Mojid, M. A., Hossain, A. Z. (2013): Conjunctive Use of Saline and Fresh Water for Irrigating Wheat (Triticum aestivum L.) at Different Growth Stages. - The Agriculturists 11: $15-23$.

[25] Morrissey, E. M., Franklin, R. B. (2015): Evolutionary history influences the salinity preference of bacterial taxa in wetland soils. - Frontiers in Microbiology 6: 1-12.

[26] Neher, D. A. (1999): Soil community composition and ecosystem processes: comparing agricultural ecosystems with natural ecosystems. - Agroforestry Systems 45(1-3): 159185.

[27] Neubauer, S. C. (2013): Ecosystem responses of a tidal freshwater marsh experiencing saltwater intrusion and altered hydrology. - Estuaries and Coasts 36(3): 491-507.

[28] Rietz, D. N., Haynes, R. J. (2003): Effects of irrigation-induced salinity and sodicity on soil microbial activity. - Soil Biology and Biochemistry 35: 845-854.

[29] Setia, R., Marschner, P., Baldock, J., Chittleborough, D., Verma, V. (2011): Relationships between carbon dioxide emission and soil properties in salt-affected landscapes. - Soil Biology and Biochemistry 43(3): 667-674.

[30] Srivastava, P. K., Gupta, M., Singh, N., Tewari, S. K. (2016): Amelioration of sodic soil for wheat cultivation using bioaugmented organic soil amendment. - Land Degradation \& Development 27(4): 1245-1254.

[31] Van der Putten, W. H., Klironomos, J. N., Wardle, D. A. (2007): Microbial ecology of biological invasions. - The ISME Journal 1(1): 28.

[32] Wang, J., Bao, J., Su, J., Li, X., Chen, G., Ma, X. (2015): Impact of inorganic nitrogen additions on microbes in biological soil crusts. - Soil Biology and Biochemistry 88: 303313. 
[33] Wang, R., Kang, Y., Wan, S., Hu, W., Liu, S., Liu, S. (2011): Salt distribution and the growth of cotton under different drip irrigation regimes in a saline area. - Agricultural Water Management 100(1): 58-69.

[34] Wang, Y. Y., Guo, D. F. (2016): Response of Soil Fungal Community Structure to Salt Vegetation Succession in the Yellow River Delta. - Current Microbiology 73(4): 595601.

[35] Xu, F., Cai, T., Yang, X., Sui, W. (2017): Soil fungal community variation by large-scale reclamation in Sanjiang plain, China. - Annals of Microbiology 67(10): 679-689.

[36] Yang, H. X., Guo, S. X., Liu, J. R. (2015): Characteristics of arbuscular mycorrhizal fungal diversity and functions in saline-alkali land. - The Journal of Applied Ecology 26(1): 311-320.

[37] Yu, Z., Wang, G., Jin, J., Liu, J., Liu, X. (2011): Soil microbial communities are affected more by land use than seasonal variation in restored grassland and cultivated Mollisols in Northeast China. - European Journal of Soil Biology 47(6): 357-363.

[38] Yuan, B. C., Li, Z. Z., Liu, H., Gao, M., Zhang, Y. Y. (2007): Microbial biomass and activity in salt affected soils under arid conditions. - Applied Soil Ecology 35(2): 319328.

[39] Zhang, P. P., Xu, S. Z., Zhang, G. J., Pu, X. Z., Wang, J., Zhang, W. F. (2019): Carbon cycle in response to residue management and fertilizer application in a cotton field in arid northwest china. - Journal of Integrative Agriculture 18(5): 1103-1119.

[40] Zhang, T., Wang, N. F., Zhang, Y. Q., Liu, H. Y., Yu, L. Y. (2015): Diversity and distribution of fungal communities in the marine sediments of Kongsfjorden, Svalbard (High Arctic). - Scientific Reports 5: 14524.

[41] Zhou, J. L., Wu, B., Wang, Y. P., Guo, X. J. (2009): Distribution and quality assessment of medium salinity groundwater in plain areas in Tarim Basin, Xinjiang. - China Rural Water Hydropower 9: 32-36. 